\title{
The Calibration of Some Stochastic Volatility Models Used in Mathematical Finance
}

\author{
Lorella Fatone1, Francesca Mariani², Maria Cristina Recchioni³, Francesco Zirilli4 \\ ${ }^{1}$ Dipartimento di Matematica e Informatica, Università di Camerino, Camerino (MC), Italy \\ ${ }^{2}$ Dipartimento di Scienze Economiche, Università degli Studi di Verona, Verona, Italy \\ ${ }^{3}$ Dipartimento di Management, Università Politecnica delle Marche, Ancona, Italy \\ "Dipartimento di Matematica "G. Castelnuovo", Università di Roma "La Sapienza", Roma, Italy \\ Email: lorella.fatone@unicam.it, francesca.mariani@univr.it, m.c.recchioni@univpm.it, zirilli@mat.uniroma1.it
}

Received 11 November 2013; revised 15 December 2013; accepted 27 December 2013

Copyright (C) 2014 Lorella Fatone et al. This is an open access article distributed under the Creative Commons Attribution License, which permits unrestricted use, distribution, and reproduction in any medium, provided the original work is properly cited. In accordance of the Creative Commons Attribution License all Copyrights () 2014 are reserved for SCIRP and the owner of the intellectual property Lorella Fatone et al. All Copyright (C) 2014 are guarded by law and by SCIRP as a guardian.

\section{Abstract}

Stochastic volatility models are used in mathematical finance to describe the dynamics of asset prices. In these models, the asset price is modeled as a stochastic process depending on time implicitly defined by a stochastic differential Equation. The volatility of the asset price itself is modeled as a stochastic process depending on time whose dynamics is described by a stochastic differential Equation. The stochastic differential Equations for the asset price and for the volatility are coupled and together with the necessary initial conditions and correlation assumptions constitute the model. Note that the stochastic volatility is not observable in the financial markets. In order to use these models, for example, to evaluate prices of derivatives on the asset or to forecast asset prices, it is necessary to calibrate them. That is, it is necessary to estimate starting from a set of data of the values of the initial volatility and of the unknown parameters that appear in the asset price/volatility dynamic Equations. These data usually are observations of the asset prices and/or of the prices of derivatives on the asset at some known times. We analyze some stochastic volatility models summarizing merits and weaknesses of each of them. We point out that these models are examples of stochastic state space models and present the main techniques used to calibrate them. A calibration problem for the Heston model is solved using the maximum likelihood method. Some numerical experiments about the calibration of the Heston model involving synthetic and real data are presented.

\section{Keywords}

Stochastic Volatility Models; Calibration 


\section{Introduction}

Stochastic processes, stochastic differential Equations and partial differential Equations are used to describe the dynamics of random phenomena. The study of their relations in mathematical physics begins with the work of Einstein [1] that establishes the relation between Brownian motion and the heat Equation. More recently random media and wave propagation in random media have been widely studied [2]. In a random medium, wave motion is studied as solution of a differential Equation with random coefficients and/or random source terms. The random coefficients and the random source terms represent the medium and usually are stochastic processes with known statistical properties. In 1973, Black and Scholes [3] introduced stochastic differential Equations in mathematical finance to model the random behavior of asset prices. The Black and Scholes asset price model can be reduced to Brownian motion with a change of variables. In particular, in the Black and Scholes model, the option pricing problem can be solved with an explicit formula (i.e. Black and Scholes formula). This model is elementary and widely used in the financial markets. However, it is known that in several circumstances the asset price dynamics model proposed by Black and Scholes in 1973 is inadequate to describe the stock prices actually observed in the financial markets. In particular, the assumption made by Black and Scholes that the asset price volatility is a constant has been criticized. To overcome this difficulty, around 1990, several stochastic volatility models of the asset price dynamics had been introduced in the theory and in the practice of mathematical finance. Models of this type are: the Hull and White model, the Stein and Stein model, the Heston model, the SABR model and many others that are not possible to mention individually. In these models the asset price and its volatility at any given time are described by random variables. The time evolution of these random variables is implicitly defined by a system of stochastic differential Equations. This system of stochastic differential Equations equipped with initial conditions and assumptions on the correlations constitutes the model. The stochastic volatility cannot be observed in the financial markets, while the asset price is observable. This means that the stochastic volatility models can be seen as stochastic state space models where the stochastic volatility Equation is the state Equation and the asset price Equation is the observation Equation. The state space models have been introduced by Kalman in 1960 [4] in the study of guidance and control problems in aerospace engineering and are widely used. The stochastic volatility models of mathematical finance depend on parameters. Moreover, the initial stochastic volatility, which cannot be observed, must be considered as a parameter. The calibration problem consists in determining the value of these parameters from a set of data. Usually the data are observations of asset prices and/or of prices of options (derivatives) having the asset as underlying at some known times. That is, calibrating a stochastic volatility model means to solve an inverse problem for a stochastic dynamical system. The use of these models in practice is based on the solution of the calibration problem. Several methods have been developed to solve the calibration problem. Let us mention some of them: generalized method of moments, maximum entropy, maximum likelihood, and statistical tests. This review paper introduces the stochastic volatility models, explains some of the calibration problems associated to them, illustrates the main calibration methods, and considers in detail as an example the problem of calibrating the Heston model with the maximum likelihood method using synthetic and real data.

\section{Background}

In their seminal work [3] Black and Scholes model the dynamics of an asset price through a stochastic differential Equation. Starting from the idea of Bachelier presented in his doctoral thesis at the École Polytechnique in Paris [5] of using Brownian motion to model the uncertainty in price behavior Black and Scholes assume that the asset price evolves according to a geometric Brownian motion with constant drift and volatility. Let $t$ be a real variable that denotes time and $S_{t}, t>0$, be a stochastic process that describes the asset price as a function of time. According to the Black and Scholes model the time dynamics of $S_{t}, t>0$, is governed by the following stochastic differential Equation:

$$
\mathrm{d} S_{t}=\mu S_{t} \mathrm{~d} t+\sigma S_{t} \mathrm{~d} W_{t}, t>0
$$

where $\mu$ is the constant drift coefficient (i.e. expected return), $\sigma$ is the constant volatility coefficient of the asset price $S_{t}, W_{t}, t>0$, is a standard Brownian motion and $\mathrm{d} W_{t}, t>0$, is its stochastic differential.

Note that the Black and Scholes model (1) is not a stochastic volatility model. The analysis of this model produces a simple and analytically convenient formula for the option prices (the Black and Scholes formula) that 
has become a standard tool to evaluate European call and put options. In 1973 Merton published a paper [6] on pricing theory enhancing the option formula derived by Black and Scholes. For their works Scholes and Merton received the Nobel Prize in Economics in 1997. Unfortunately Black died in 1995. Although the Black and Scholes model is adequate to describe the asset price dynamics in short time intervals, when we consider long time series of option prices the model does not capture some characteristic features of the volatility implied by the observed option prices known as volatility smile and skew. In particular given the observed option prices the volatilities implied by the Black and Scholes formula for options with different strike and maturity are different. This is in contrast with the assumption of the Black and Scholes model that the volatility is a constant. The time series of asset and option prices observed in the financial markets show that the volatility of the asset price is a non-constant quantity that tends to return over time to a long-term mean level, that is the behavior of the volatility can be described satisfactorily by a stochastic mean-reverting process.

\section{The Stochastic Volatility Models}

The stochastic volatility models try to overcome this inadequacy of the Black and Scholes model assuming that also the volatility is modeled as a stochastic process whose dynamics is described by a stochastic differential Equation. The presence of the volatility term in the Equation that describes the dynamics of the asset price couples the differential Equations for the asset price and for its stochastic volatility generating a system of two stochastic differential Equations. This is the simplest setting of a stochastic volatility model in mathematical finance. These Equations can be interpreted as a model where the asset price propagates in a random medium described by the stochastic volatility. That is stochastic volatility models are somehow similar to the models of propagation in random media used in mathematical physics.

The first authors that have suggested the use of a stochastic volatility model in mathematical finance are Hull and White in 1987 [7]. The Hull and White model describes the asset price dynamics as follows:

$$
\begin{aligned}
& \mathrm{d} S_{t}=\mu S_{t} \mathrm{~d} t+\sqrt{v_{t}} S_{t} \mathrm{~d} W_{t}, t>0, \\
& \mathrm{~d} v_{t}=\theta v_{t} \mathrm{~d} t+\xi v_{t} \mathrm{~d} B_{t}, \quad t>0 .
\end{aligned}
$$

where $v_{t}, t>0$, denotes the stochastic variance of the asset price $S_{t}, t>0$, at time $t$, i.e. the volatility $\sigma_{t}, t>0$, is given by $\sigma_{t}=\sqrt{v_{t}}, t>0, \theta, \xi$ are, respectively, the drift and the volatility coefficients of the variance $v_{t}$ and $\mu$ is the drift coefficient of the asset price. The quantities $\theta, \xi, \mu$ are real constants and $\theta<0$. The stochastic processes $W_{t}, B_{t}, t>0$, are standard Brownian motions with correlation coefficient $\rho,-1<\rho<1$ and $\mathrm{d} W_{t}, \mathrm{~d} B_{t}, t>0$, are their stochastic differentials. Note that in (2) the stochastic variance is lognormally distributed and that when $\theta<$ 0 , we have a zero-reverting process, i.e. the variance tends to return over time to a long-term zero level. When $\rho$ $=0$ using a second order Taylor expansion with base point $\xi=0$ Hull and White derived an explicit formula for the option prices whose underlying asset price dynamics is described by (2). Moreover the formula for the option prices was used only when $\theta$ is zero. The biggest weakness of this formula is that it is accurate when the variance is almost non-stochastic. In the general case (i.e. $\rho \neq 0, \theta \neq 0$ ) the option pricing problem in the Hull and White model is solved numerically. Note that the assumption $\rho=0$ is not supported by observations of asset prices in the financial markets. In fact the observed prices show that usually $\rho$ is negative.

The Stein and Stein model [8] assumes that:

$$
\begin{aligned}
& \mathrm{d} S_{t}=\mu S_{t} \mathrm{~d} t+\sigma_{t} S_{t} \mathrm{~d} W_{t}, \quad t>0, \\
& \mathrm{~d} \sigma_{t}=\eta\left(\theta-\sigma_{t}\right) \mathrm{d} t+\xi \mathrm{d} B_{t}, t>0,
\end{aligned}
$$

where $\mu, \eta, \theta, \xi$ are real constants such that $\eta>0$ and $W_{t}, B_{t}, t>0$, are standard Brownian motions with correlation $\rho,-1<\rho<1$.

In the Stein and Stein model when $\eta>0$, the stochastic volatility $\sigma_{t}, t>0$ follows a mean-reverting Ornstein-Uhlenbeck process. Note that in this model the volatility can become negative. The Stein and Stein model overcomes the difficulty of the Hull and White model to provide effective option pricing formulae providing a closed-form formula for the prices of the options on a underlying whose price dynamics is described by (3). This formula is deduced under the assumption $\rho=0$.

A very popular stochastic volatility model is the Heston model [9]. The Heston model assumes that the asset price $S_{t}, t>0$, and its stochastic variance $v_{t}, t>0$, satisfy the following Equations: 


$$
\begin{aligned}
& \mathrm{d} S_{t}=\mu S_{t} \mathrm{~d} t+\sqrt{v_{t}} S_{t} \mathrm{~d} W_{t}, \quad t>0, \\
& \mathrm{~d} v_{t}=\eta\left(\theta-v_{t}\right) \mathrm{d} t+\xi \sqrt{v_{t}} \mathrm{~d} B_{t}, t>0,
\end{aligned}
$$

where $\theta$ is the long-term average of $v_{t}, t>0, \eta>0$ is the rate of mean-reversion, $\xi$ is the volatility of volatility coefficient. The quantities $\mu, \theta, \eta, \xi$ are real constants, $\eta>0$, and $W_{t}, B_{t}, t>0$, are standard Brownian motions with correlation coefficient $\rho,-1<\rho<1$. In the Heston model the variance follows a Cox-Ingersoll-Ross (CIR) process [10]. The popularity of the Heston model is due to the fact that, when $-1<\rho<1$, it is possible to deduce a closed form formula for the price of an European call (put) option whose underlying asset price and its stochastic volatility satisfy Equations (4). The formulae for the option prices are expressed as integrals of explicitly known integrands. These integrals must be evaluated numerically.

Note that the stochastic volatility models mentioned above assume that the asset price follows the Black and Scholes Equation (1) with stochastic volatility, the difference between them consists in the stochastic process that governs the dynamics of the volatility.

Since the pioneering work of the previous authors many other stochastic volatility models have been introduced in mathematical finance. It is not possible to mention all of them. For the convenience of the reader we just mention one of them: the SABR model [11]. The name SABR is the acronym for "Stochastic Alpha, Beta, Rho" in fact $\alpha, \beta, \rho$ are the parameters of the model. The SABR model is widely used to price derivatives on interest rates and currency exchange rates. In the SABR model the time evolution of the asset price and of its stochastic volatility satisfy the following system of stochastic differential Equations:

$$
\begin{aligned}
& \mathrm{d} S_{t}=\sigma_{t} S_{t}^{\beta} \mathrm{d} W_{t}, \quad t>0, \\
& \mathrm{~d} \sigma_{t}=\alpha \sigma_{t} \mathrm{~d} B_{t}, \quad t>0,
\end{aligned}
$$

where $\alpha$ and $\beta$ are real constants such that $\alpha>0,0 \leq \beta \leq 1$ and $W_{t}, B_{t}, t>0$, are standard Brownian motions with correlation coefficient $\rho,-1<\rho<1$. The parameter $\alpha$ is called volatility of volatility coefficient. Note that the SABR model is a stochastic version of the constant elasticity of variance (CEV) model [12] with skewness parameter $\beta$ and that it reduces to the CEV model when $\alpha=0$. The stochastic volatility models are systems of stochastic differential Equations and to determine their solution they must be equipped with initial conditions assigned, for example, at time $t=0$.

Unlike the asset price, in the financial markets the volatility cannot be observed. That is the stochastic volatility models are systems of stochastic differential Equations for two dependent variables, one of these variables (the asset price) is observable the other variable (the stochastic volatility or variance) is not observable. If we interpret the stochastic volatility (or variance) as a state variable and the asset price as an observation variable, the stochastic volatility models can be seen as stochastic state space models of a continuous-time dynamic system. In fact the state (or transition) Equation of the model will be the stochastic differential Equation for the volatility (or variance) and the observation (or measurement) Equation of the model will be the Equation for the asset price. Kalman [4] for a special class of these models has developed a method to estimate the unknown parameters and the state variable from a time series of values of the observation variable. This technique is known as Kalman filter or linear quadratic estimation (LQE) algorithm. Today state space models and Kalman filters are widely used in science and engineering to study time series of data and to forecast future events based on past data [13].

\section{Calibration Methods}

The problem of identifying the parameters of stochastic volatility models starting from observed data is an inverse problem known in mathematical finance as "calibration problem". There are many calibration problems associated to stochastic volatility models depending on the data used in the calibration process. Usually the data of a calibration problem are a time series of asset prices and/or of option prices. Note that when option prices are used as data of the calibration problem it is necessary to compute their theoretical value in the stochastic volatility model considered as a function of the values assigned to the parameters. This theoretical value is defined as the expected value of the discounted payoff of the option under a risk neutral probability measure. The stochastic volatility models discussed previously admit infinitely many risk neutral measures. These measures are parameterized by a real parameter called risk premium parameter, also known as market price of volatility risk. 
Therefore the use of option prices as data for the calibration problem introduces an extra parameter to estimate: the risk premium parameter. Note that inverse problems for stochastic state space models are widely studied in many branches of science and engineering. However, the use of option prices as data is a feature that characterizes these problem in the context of mathematical finance.

There are several calibration methods, and we discuss briefly the following ones: the generalized method of moments, the maximum entropy method, the maximum likelihood method and the method of statistical tests.

In the generalized method of moments [14] the unknown parameters solution of the calibration problem are those that minimize the difference between the moments defined by the model of certain variables (i.e. theoretical moments) and the same moments estimated from the data (i.e. sample moments). In order to use this method it is necessary to know the analytic expression of the (theoretical) moments or to compute them numerically. Note that the moments of the asset price variable are the natural candidates for the application of the method of moments. However other choices are possible. For example in [15] the authors starting from the observations of the asset prices at discrete times apply the generalized method of moments to calibrate square root continuous time stochastic volatility models.

They use as theoretical moments the moments of the integrated volatility conditioned to the observations of the asset price and as sample moments the sample moments of the realized volatility.

The maximum entropy method [16] has his origin in statistical mechanics in the study of the properties of thermodynamic systems at equilibrium. The idea of the maximum entropy principle, which is the basis of the homonymous method for the calibration of stochastic volatility models, is to find the values of the unknown parameters of the model as those that maximize the entropy associated to the probability distribution of the asset price and of its stochastic volatility conditioned to the observations. The entropy of a probability distribution measures the randomness in the probability distribution [17] and in the context of statistical mechanics coincides with the thermodynamic entropy. Many authors have developed variants of the maximum entropy method, in particular in [18] the optimal parameters solution of the calibration problem are found as those that minimize the relative entropy between the probability distribution associated to the model and a reference distribution.

The maximum likelihood method determines the unknown parameters of the stochastic volatility model as the parameter values that maximize a likelihood function associated to the data. The likelihood function is a function that measures the probability of obtaining the observed data as a function of the parameter values. The evaluation of the likelihood function usually requires the knowledge of the (joint) probability density function of the asset price and of its volatility. When the model used is a stochastic volatility model one of the variables (i.e. the volatility) is not observable so that the use of filtering techniques is necessary to evaluate the likelihood function. Note that given a stochastic volatility model there are several possibilities of defining a meaningful likelihood function. In [19] a closed-form approximation of a likelihood function associated to the Heston model is derived using a series expansion. The approximate likelihood function is maximized to find the parameters of the Heston model. In 2006 Bates [20] has found an approximation of a likelihood function associated to exponentially affine stochastic processes. This approximation is built starting from the computation of the inverse Fourier transform of the joint characteristic function of the asset price and its stochastic volatility on the observed asset prices. The parameters estimated maximizing the likelihood function are used to forecast the unknown volatility values. In [21] the joint probability density function of the Heston model conditioned to the observations is obtained as solution of a sequence of initial value problems for the Fokker-Planck Equation of the model (i.e. as solution of the Kushner Equation). The likelihood function of the parameters can be expressed through integrals of this transition probability density function. The main feature that makes the calibration problem studied in [21] interesting is the use as data of observations of asset prices and of option prices. The use of this set of data guarantees that the parameters obtained maximizing the likelihood function determine a good match between theoretical option prices and option prices observed in the past and produce satisfactory forecast values of asset and option prices.

Note that the maximum likelihood method is statistically more efficient than the generalized method of moments when this last method is applied using a finite number of moments. In fact the probability density function used in the likelihood function contains more "information" about the model than the "information" contained in a finite number of moments of a variable of the model. However when the evaluation of the likelihood function is difficult or computationally expensive the generalized method of moments can be a valid alternative to the maximum likelihood method.

The methods described previously provide estimates for the values of the unknown parameters of the models 
studied but do not associate a statistical significance level to them. The use of statistical hypothesis testing can overcome this weakness of the previous methods. In this case, the null hypothesis of a statistical test states that the values of the model parameters are equal to the values estimated with one of the methods mentioned previously. Recall that the significance level is the maximum probability of rejecting the null hypothesis when the null hypothesis is true. Starting from the data sample available of the observed asset and/or option prices, the statistical test technique can be used to test the null hypothesis with a given statistical significance level. The idea of using statistical tests to solve calibration problems for stochastic dynamical systems is a very natural one. In [22] a statistical test procedure to calibrate the Black and Scholes model is presented. The procedure makes use of elementary statistical tests, that is: the Student's $\mathrm{T}$ and the $\chi^{2}$ tests [23]. The data used to calibrate the model are the observations on a discrete set of time values of the asset price. Note that when the asset price dynamics follows the Black and Scholes model the asset price log-return increments (i.e. the difference between the asset price log-returns evaluated at different times) obtained when the observation times are equally spaced time values are independent identically distributed Gaussian random variables. This implies that the calibration can be done using the elementary statistical tests already mentioned. In more general contexts when stochastic volatility models are considered it is necessary to build ad hoc statistical tests. For example in [24] two statistical tests to calibrate the normal SABR model (with statistical significance level) in correspondence of two different set of data are presented. These statistical tests are based on some new formulae for the moments of the normal SABR model variables and make use of numerical methods.

For the convenience of the reader Table 1 summarizes the calibration methods discussed in this Section and their properties.

\section{An Example: The Calibration of the Heston Model}

Let us consider the problem of calibrating the Heston model using the maximum likelihood method. As data of the calibration problem we use a time series of asset and option prices. This problem is an example of calibration of a stochastic volatility model and its solution shows the concrete possibilities of the calibration methods discussed in Section 4. A similar problem has been studied in [21].

Let us begin completing the Heston model dynamic Equation (4) with the following initial conditions:

$$
S_{o}=\tilde{S}_{o}, v_{o}=\tilde{v}_{o}
$$

where $\tilde{S}_{o}, \tilde{v}_{o}$ are given random variables. We assume that $\tilde{S}_{o}, \tilde{V}_{o}$ are concentrated in a point with probability one, for simplicity, we identify $\tilde{S}_{o}, \tilde{v}_{o}$ with the points where they are concentrated and we assume $\tilde{S}_{o}, \tilde{v}_{o}>0$.

Table 1. Summary of calibration methods and of their properties.

\begin{tabular}{|c|c|c|c|}
\hline & Calibration method & Foundation of the method & Comment \\
\hline 1 & $\begin{array}{l}\text { Generalized method } \\
\text { of moments }\end{array}$ & $\begin{array}{l}\text { The unknown parameters are obtained minimizing } \\
\text { the difference between the moments defined by the } \\
\text { model of some random variable and the same } \\
\text { moments estimated from the data. }\end{array}$ & $\begin{array}{l}\text { This is the most popular calibration method. Option } \\
\text { prices can be seen as moments of random variables. } \\
\text { In this case the calibration can be reduced to the least } \\
\text { squares fit of theoretical and observed option prices. }\end{array}$ \\
\hline 2 & Maximum Entropy & $\begin{array}{l}\text { The unknown parameters are obtained maximizing } \\
\text { the entropy of the probability distribution of the } \\
\text { asset price and of its stochastic volatility conditioned } \\
\text { to the observations. The entropy is a measure of the } \\
\text { randomness of a probability distribution. }\end{array}$ & $\begin{array}{l}\text { This method is based on ideas taken from statistical } \\
\text { mechanics. Both option prices and asset prices can be } \\
\text { used as data. The entropy function can be substituted } \\
\text { with an approximate entropy function to save } \\
\text { computation. }\end{array}$ \\
\hline 3 & Maximum Likelihood & $\begin{array}{l}\text { The unknown parameters are obtained maximizing } \\
\text { a likelihood function associated to the data. The } \\
\text { likelihood function measures the probability of } \\
\text { obtaining the observed data as a function of the } \\
\text { parameter values. }\end{array}$ & $\begin{array}{l}\text { Both option prices and asset prices can be used as } \\
\text { data. The method exploits the fact that the joint } \\
\text { probability distribution of the state variables of the } \\
\text { models considered admits a simple integral } \\
\text { representation formula. }\end{array}$ \\
\hline 4 & Statistical Tests & $\begin{array}{l}\text { The unknown parameters are determined using } \\
\text { statistical hypothesis testing. Ad hoc statistical } \\
\text { tests are developed to exploit the available data. }\end{array}$ & $\begin{array}{l}\text { This method associates to the values of the parameters } \\
\text { determined as solution of the calibration problem a } \\
\text { statistical confidence. The ad hoc statistical tests built } \\
\text { are based on sampling suitable random variables using } \\
\text { numerical simulation. }\end{array}$ \\
\hline
\end{tabular}


Recall that in the financial markets the initial asset price $\tilde{S}_{o}$ can be observed while the initial stochastic variance $\tilde{v}_{o}$ cannot be observed and must be regarded as a parameter of the model. This means that the Heston model parameters that must be evaluated from the data in the calibration problem are:

$\mu, \eta, \theta, \xi, \rho, \tilde{v}_{o}$. When we consider option prices as data of the calibration problem to the previous parameters we must add the risk premium parameter $\lambda$. In fact the option prices are evaluated with respect to the risk neutral measure. This is the probability measure associated to the Heston model where $\eta$ and $\theta$ are substituted respectively with $\eta^{*}=\eta+\lambda, \theta^{*}=\frac{\eta \theta}{\eta+\lambda}$. Let $R^{7}$ be the seven dimensional real Euclidean space, the unknown of the calibration problem is the vector $\underline{\Theta}=\left(\mu, \eta, \theta, \xi, \rho, \tilde{v}_{o}, \lambda\right)^{\mathrm{T}} \in R^{7}$ where T denotes the transpose operator. Elementary considerations suggest that $\underline{\Theta}$ must belong to the following set:

$$
M=\left\{\underline{\Theta}=\left(\mu, \eta, \theta, \xi, \rho, \tilde{v}_{o}, \lambda\right)^{\mathrm{T}} \in \mathrm{R}^{7} \mid \eta>0, \xi>0, \theta>0, \frac{2 \eta \theta}{\xi^{2}} \geq 1,-1 \leq \rho \leq 1, \tilde{v}_{o}>0, \lambda>-\eta\right\} .
$$

The condition $\frac{2 \eta \theta}{\xi^{2}} \geq 1$ is imposed in order to guarantee that when $\tilde{v}_{o}>0$ with probability one from Equations (4) follows that $v_{t}$ is positive with probability one for $t>0$.

The data of the calibration problem studied are:

1) The observation times $0=t_{0}<t_{1}<t_{2}<\cdots<t_{n}<+\infty$,

2) The observed asset price $\tilde{S}_{i}$ at time $t=t_{i}, i=0,1, \cdots, n$,

3) The observed option price $\tilde{C}_{i}$ at time $t=t_{i}, i=0,1, \cdots, n$.

The option prices are the prices of a European call option on the asset whose price is described by the Heston model with (known) maturity time $T>t_{n}$ and strike price $E>0$.

We assume that the observation times and the observed asset prices are not affected by observation errors and that the asset prices are described by the Heston model, while for $i=1,2, \cdots, n$ the $i$-th observed option price when compared to the option price of the Heston model is affected by an additive observation error that we assume to be normally distributed with mean zero and known variance $\phi_{i}$.

Let $p\left(S, v, t, S^{\prime}, v^{\prime}, t^{\prime}\right), S, S^{\prime}, v, v^{\prime}, t, t^{\prime}>0, t-t^{\prime} \geq 0$, be the joint probability density function of the stochastic processes implicitely defined by (4), (6), that is let $p\left(S, v, t, S^{\prime}, v^{\prime}, t^{\prime}\right)$ be the probability density function of having $S_{t}=S, v_{t}=v$ given the fact that we have $S_{t^{\prime}}=S^{\prime}, v_{t^{\prime}}=v^{\prime}, t>t^{\prime} \geq 0$. Moreover let $C(S, v, t, E, T, \underline{\Theta})$ be the price at time $t$ when we have $S_{t}=S, v_{t}=v$ in the Heston model of parameters $\underline{\Theta}$ of a European call option with maturity time $T>t$ and strike price $E$. In the theoretical option price $C(\bar{S}, v, t, E, T, \underline{\Theta})$ we use as discount factor $\mu$ instead of the risk free interest rate $r$ (i.e. we assume $\mu=r$ ). This choice simplifies a little bit the calibration problem. The probability density function $p$ and the call option price $C$ can be written as one dimensional integrals of known integrand functions (see, for example [21]). To keep the exposition simple we omit these formulae.

In the formulation of the calibration problem that follows we use the joint probability density function of the variables $S_{t}, v_{t}, t>0$, of the Heston model conditioned to the observations $\tilde{S}_{i}, \tilde{C}_{i}$ made at the times $t_{i}$ such that $t_{i}<t, i=0,1, \cdots, n$. This probability density function is a piecewise solution of the forward Kolmogorov Equation associated to the Heston model. That is the transition probability density function conditioned to the observations between two consecutive observation times $t_{i}, t_{i+1}, i=0,1, \cdots, n$, (we define $t_{n+1}=+\infty$ ) is the solution of the forward Kolmogorov Equation that satisfies a suitable initial condition at time $t=t_{i}, i=0,1, \cdots, n$. The initial condition imposed at time $t=t_{i}$ depends from the observations made up to time $t_{i}, i=0,1, \cdots, n$, and on the assumptions made about the observation errors (see [21]). For $i=0,1, \cdots, n$ let $p_{i}(S, v, t \mid \underline{\Theta}$ ) be the joint probability density function conditioned to the observations made up to time $t$ when $t_{i}<t \leq t_{i+1}$. The functions $p_{i}, i=0,1, \cdots, n$, can be expressed using integral representation formulae involving the transition probability function $p$ (see [21]). To keep the exposition simple we omit these formulae here.

Let us define the likelihood function $F(\underline{\Theta}), \underline{\Theta} \in M$, used in the formulation the calibration problem, that is:

$$
F(\underline{\Theta})=\sum_{i=0}^{n-1} \log \left[\int_{0}^{+\infty} \mathrm{d} v p_{i}\left(\tilde{S}_{i+1}, v, t_{i+1} \mid \underline{\Theta}\right) \pi_{1}\left(\tilde{S}_{i+1}, v, t_{i+1} \mid \underline{\Theta}\right)+\log \left[\int_{0}^{+\infty} \mathrm{d} v p_{0}\left(\tilde{S}_{0}, v, t_{0} \mid \underline{\Theta}\right) \cdot \pi_{1}\left(\tilde{S}_{0}, v, t_{0} \mid \underline{\Theta}\right)\right],\right.
$$


where

$$
\pi_{1}\left(\tilde{S}_{i}, v, t_{i} \mid \underline{\Theta}\right)=\frac{1}{\sqrt{2 \pi \phi}} \mathrm{e}^{-\frac{1}{2 \phi_{i}^{2}}\left(\tilde{C}_{i}-C\left(\tilde{S}_{i}, v, t_{i}, E, T, \underline{\Theta}\right)\right)^{2}}, i=0,1, \cdots, n
$$

It is easy to see that the choices of $\underline{\Theta} \in M$ that make big the function $F(\underline{\Theta})$ make big the probability of doing the observations $\tilde{S}_{i}, \tilde{C}_{i}$ actually done at time $t=t_{i}, i=0,1, \cdots, n$. This fact justifies the name likelihood function given to the function $F(\underline{\Theta}), \underline{\Theta} \in M$, defined in (8), (9). Note that the choice of the function $F(\underline{\Theta}), \underline{\Theta} \in M$, made in (8), (9) is only one possible choice of likelihood function, there are many other ways of defining legitimate likelihood functions.

The calibration problem for the Heston model is formulated as follows:

$$
\max F(\underline{\Theta}) . \underline{\Theta} \in M
$$

This is a constrained optimization problem that must be solved numerically. The objective function of the optimization problem $F(\underline{\Theta}), \underline{\Theta} \in M$, contains several integrals that must be evaluated numerically. That is in the numerical solution of problem (10) the function evaluation can be computationally "expansive”. However since the numerical evaluation of integrals is highly parallelizable when needed parallel computing can be easily used to speed up the evaluation of the function $F(\underline{\Theta}), \underline{\Theta} \in M$. The constraints contained in $M$ are very simple and are easy to handle numerically. In the numerical experience presented below we solve the calibration problem (10) using a variable metric steepest ascent algorithm.

Let us present two numerical experiments using synthetic and real data.

Experiment 1: Calibration of the Heston model using synthetic data. We consider a "year" made of 252.5 trading days, a temporal interval of 36 consecutive trading days and $n=9$ observation times in this time period. We choose $t_{0}=0$, and $t_{i}=t_{i-1}+\Delta, \quad i=1,2, \cdots, 9$, where $\Delta=\frac{4}{252.5}$. The vector $\underline{\Theta}$ used to generate one trajectory of the Heston model is:

$$
\begin{aligned}
\underline{\Theta} & =\left(\mu, \gamma, \varepsilon, \theta, \rho, \tilde{v}_{o}, \lambda\right)^{\mathrm{T}}=\underline{\tilde{\Theta}} \\
& =(0.026,5.94,0.306,0.01159,-0.576,0.5,0)^{\mathrm{T}} .
\end{aligned}
$$

We have chosen $\tilde{S}_{o}=1, \phi_{i}=10^{-4}, \quad i=0,1, \cdots, 9$. In Table 1 we have defined $\tilde{x}_{i}=\log \frac{\tilde{S}_{i}}{\tilde{S}_{o}}, i=0,1, \cdots, 9$, and the notation $\mathrm{e} X$ means $10^{X}$. Table 2 shows the data generated.

Table 2. Time series of simulated data.

\begin{tabular}{cccc}
\hline$i$ & $t_{i}$ (year) & $\tilde{x}_{i}$ & $\tilde{C}_{i}$ \\
\hline 0 & 0 & 0 & 0.5099 \\
1 & $4 / 252.5$ & $1.235 e^{-1}$ & 0.6410 \\
2 & $8 / 252.5$ & $6.893 e-2$ & 0.5804 \\
3 & $12 / 252.5$ & $-2.9809 e-2$ & 0.4791 \\
4 & $16 / 252.5$ & $1.2049 e-2$ & 0.5201 \\
5 & $20 / 252.5$ & $4.4983 e-2$ & 0.5538 \\
6 & $24 / 252.5$ & $-7.7662 e-4$ & 0.5065 \\
7 & $28 / 252.5$ & $-1.3411 e^{-2}$ & 0.4936 \\
8 & $32 / 252.5$ & $-1.2461 e^{-1}$ & 0.3894 \\
9 & $36 / 252.5$ & $-7.10141 e^{-2}$ & 0.4377 \\
\hline
\end{tabular}


We have $F(\underline{\tilde{\Theta}}) \cong 43.15$. The initial guess of the optimization method is chosen equal to $\underline{\Theta}^{0}=(0.5,9.22,0.15,0.0067,-0.634,0.3,0.01) \in M$. We have $\left\|\underline{\tilde{\Theta}}-\underline{\Theta}^{0}\right\| /\|\underline{\tilde{\Theta}}\| \approx 0.398, F\left(\underline{\Theta}^{0}\right)=-979.1$. Let $\underline{\Theta}{ }^{*}$ be the solution of the calibration problem (10) obtained using the variable metric steepest ascent algorithm, we have $F\left(\underline{\Theta}^{*}\right)=43.17$ and $\|\underline{\tilde{\Theta}}-\underline{\Theta}\| /\|\underline{\tilde{\Theta}}\| \approx 0.041$.

Experiment 2: Analysis of the S \& P 500 index. We analyze the daily closing values of the US S\&P 500 index and the corresponding bid prices of a European call option on the US S\&P 500 index with maturity date December 16, 2005 and strike price $E=1200$ during the period of about four months going from January 3, 2005 to May 11, 2005. Due to the number of data actually available in 2005 the time unit is a year made of 253 trading days. We choose as time $t=0$ the day January 3, 2005. We consider 81 windows of 10 consecutive daily observation times. In particular the observation times are chosen as $t_{i, j}=(i+j) / 253, i=0,1, \cdots, 9, j=0,1, \cdots, 80$, that is in each window we consider daily observations and when we move from a window to the next one we discard the observations made in the first day of the window and we add the next daily observations as last observations to build the new window. The calibration problems procedure, applied to the eighty-one windows considered provides eighty-one values of the parameter vector $\underline{\Theta}$ of the Heston model. The parameter vectors obtained from the calibration problems are substantially independent from the data window considered. Finally for $j=0,1, \cdots, 80$ we use the parameter vector obtained as solution of the calibration problem using the data of the $j$-th window to forecast using a filtration method (see [21]) the value of the S\&P 500 index and the bid price of the option the day next to the last observation time of the $j$-th window. In the eighty-one calibration and forecasting problems solved the mean relative error made in the forecasts of the next day S\&P 500 index value and option bid price are 0.0063 and 0.0785 respectively.

From the results obtained in Experiment 1 and 2 we can conclude that the validity of the Heston model to interpret financial data and the quality of the calibration procedure used are confirmed.

\section{Future Research Directions}

The stochastic volatility models described in the previous sections can be generalized in many different ways. For example multiscale versions of stochastic volatility models are used in finance to model commodity prices or to evaluate insurance premia. These multiscale models introduce one or more extra stochastic volatility terms in the model. The multiscale version of a model usually increases the number of parameters to estimate making the calibration problem more difficult and more demanding computationally than the calibration problem for the original model. In order to overcome this difficulty it is necessary to develop ad-hoc techniques to simplify the computation or it is possible to make use of parallel computing. In [25] and in [26], independently, the authors introduce a multiscale stochastic volatility model that generalizes the Heston model. In the multiscale Heston model two stochastic volatilities are considered. In [26] an extension of the maximum likelihood method has been used to calibrate the multiscale Heston model. This calibration is based on the reduction of the dimensionality of the integrals that must be computed to evaluate the likelihood function. This reduction of the dimensionality of the integrals is obtained computing analytically some of the integrals that appear in the likelihood function. On the other hand in more general situations parallel computing and GPU computing can be used to speed up the evaluation of the likelihood function.

Note that the calibration methods described in the previous section have been applied only to special classes of stochastic volatility models. For example in [20] and in [21] the authors limit themselves to the calibration of exponentially affine stochastic volatility models because for these models there exists an explicit formula (involving integrals) for the joint characteristic function of asset price and its stochastic volatility. It should be interesting and useful to apply the calibration methods to the study of more general classes of models.

Finally cross fertilization between different research areas is possible. In fact, for example, the methods used to solve inverse problems for state space models outside mathematical finance, such as particle filtering, can be transferred in financial mathematics and used to calibrate stochastic volatility models [27].

\section{Conclusion}

The problem of calibrating stochastic volatility models is crucial for their use in practical situations. The methods described in the previous sections provide different approaches to solve this calibration problem. These 
methods find the unknown parameters of the model as solution of optimization problems and differ in the choice of the objective function of the optimization problem considered. The method that uses statistical tests is the only one that associates a statistical significance to the solution found. However, the choice of the hypothesis to be tested in the solution of the calibration problem may be difficult when models with several parameters (five, six parameters or more) are employed. A way to overcome this difficulty consists in using a different calibration method (i.e.: generalized method of moments, maximum entropy, ...) to determine the solution of the calibration problem and in using the solution found to formulate the hypothesis to be tested. To this hypothesis is given a statistical significance using the statistical test method. We can conclude that the calibration problems for the stochastic volatility models developed in the nineties have been solved satisfactorily. The dramatic change of financial markets and of computing technologies announces the development of new, more sophisticated, stochastic volatility models whose calibration will pose new challenges. We expect that new problems and ideas will arise from considering these new models and the specific features that derive from their origin in finance.

\section{References}

[1] Einstein, A. (1905) Über die von der molekularkinetischen theorie der Wärme geforderte Bewegung von in ruhenden Flüssigkeiten suspendierten Teilchen. Annalen der Physik, 322, 549-560. http://dx.doi.org/10.1002/andp.19053220806

[2] Keller, J.B. (1964) Wave propagation in random media. Proceedings of Symposia in Applied Mathematics, 13, 227246.

[3] Black, F. and Scholes, M. (1973) The pricing of options and corporate liabilities. Journal of Political Economy, 81, 637-659. http://dx.doi.org/10.1086/260062

[4] Kalman, R.E. (1960) A new approach to linear filtering and prediction problems. Journal of Basic EngineeringTransactions of the ASME, Series D, 85, 35-45. http://dx.doi.org/10.1115/1.3662552

[5] Bachelier, L. (1900) Théorie de la speculation. Annales Scientifiques de l'École Normale Supérieure, 3, 21-86.

[6] Merton, R.C. (1973) Theory of rational option pricing. Bell Journal of Economics and Management Science, 4, 141183. http://dx.doi.org/10.2307/3003143

[7] Hull, J. and White, A. (1987) The pricing of options on assets with stochastic volatilities. Journal of Finance, 42, 281300. http://dx.doi.org/10.1111/j.1540-6261.1987.tb02568.x

[8] Stein, E.M. and Stein, J.C. (1991) Stock price distribution with stochastic volatility: An analytic approach. Review of Financial Studies, 4, 727-752. http://dx.doi.org/10.1093/rfs/4.4.727

[9] Heston, S.L. (1993) A closed-form solution for options with stochastic volatility with applications to bond and currency options. The Review of Financial Studies, 6, 327-343. http://dx.doi.org/10.1093/rfs/6.2.327

[10] Cox, J.C., Ingersoll, J.E. and Ross, S.A. (1985) A theory of the term structure of interest rates. Econometrica, 53, 385407. http://dx.doi.org/10.2307/1911242

[11] Hagan, P.S., Kumar, D., Lesniewski, A.D. and Woodward, E. (2002) Managing smile risk. Wilmott Magazine, 2002, 84-108. http://www.wilmott.com/pdfs/021118 smile.pdf

[12] Cox, J. (1975) Notes on option pricing I: Constant elasticity of diffusions. Unpublished Draft, Stanford University.

[13] Harvey, A.C., Koopman, S.J. and Shephard, N. (2004) State space and unobserved component models: Theory and applications. Cambridge University Press, Cambridge.

[14] Hansen, L.P. (1982) Large sample properties of generalized method of moments estimators. Ecometrica, 50, 10291054.

[15] Bollerslev, T. and Zhou, H. (2002) Estimating stochastic volatility diffusion using conditional moments of integrated volatility. Journal of Econometrics, 109, 33-65. http://dx.doi.org/10.1016/S0304-4076(01)00141-5

[16] Jaynes, E.T. (1957) Information theory and statistical mechanics. Physical Review, 106, 620-630. http://dx.doi.org/10.1103/PhysRev.106.620

[17] Shannon, C.E. (1948) A mathematical theory of communication. Bell System Technical Journal, 27, 379-423. http://dx.doi.org/10.1002/j.1538-7305.1948.tb01338.x

[18] Avellaneda, M. (1998) Minimum-relative-entropy calibration of asset pricing models. International Journal of Theoretical and Applied Finance, 1, 447-472. http://dx.doi.org/10.1142/S0219024998000242

[19] Ait-Sahalia, Y. and Kimmel, R. (2007) Maximum likelihood estimation of stochastic volatility models. Journal of Financial Economics, 83, 413-452. http://dx.doi.org/10.1016/j.jfineco.2005.10.006

[20] Bates, D.S. (2006) Maximum likelihood estimation of latent affine processes. The Review of Financial Studies, 19, 909-965. http://dx.doi.org/10.1016/j.jfineco.2005.10.006 
[21] Mariani, F., Pacelli, G. and Zirilli, F. (2008) Maximum likelihood estimation of the Heston stochastic volatility model using asset and option prices: An application of nonlinear filtering theory. Optimization Letters, 2, 177-222. http://dx.doi.org/10.1007/s11590-007-0052-7

[22] Fatone, L., Mariani, F., Recchioni, M.C. and Zirilli, F. (2012) The use of statistical tests to calibrate the Black-Scholes asset dynamics model applied to pricing options with uncertain volatility. Journal of Probability and Statistics, 2012, Article ID: 931609.

[23] Johnson, R.A. and Bhattacharyya, G.K. (2006) Statistics: Principles and methods. 5th Edition, John Wiley \& Sons, New York.

[24] Fatone, L., Mariani, F., Recchioni, M.C. and Zirilli, F. (2013) The use of statistical tests to calibrate the normal SABR model. Journal of Inverse and Ill-Posed Problems, 21, 59-84. http://dx.doi.org/10.1515/jip-2012-0093

[25] Christoffersen, P., Heston, S. and Jacobs, K. (2009) The shape and term structure of the index option smirk: Why multifactor stochastic volatility models work so well. Management Science, 55, 1914-1932. http://dx.doi.org/10.1287/mnsc.1090.1065

[26] Fatone, L., Mariani, F., Recchioni, M.C. and Zirilli, F. (2009) An explicitly solvable multi-scale stochastic volatility model: Option pricing and calibration problems. Journal of Futures Markets, 29, 862-893. http://dx.doi.org/10.1002/fut.20390

[27] Mergner, S. (2009) Application of state space models in finance: An empirical analysis of the time-varying relationship between macroeconomics, fundamentals and Pan-European industry portfolios. Universitätsverlag Göttingen, Göttingen. 